\title{
DISCONTINUOUS SOLUTIONS OF NEUTRAL FUNCTIONAL DIFFERENTIAL EQUATIONS
}

\author{
J. C. F. DE Oliveira and L. Fichmann
}

\begin{abstract}
The fundamental theory of existence, uniqueness and continuous differentiability of $L^{p}$-solutions for Neutral Functional Differential Equations is presented. Also, the spectrum of the solution operator of general autonomous linear NFDFs is described. Finally; an extension of Hartman Grobman Theorem on local conjugacy near a hyperbolic equilibrium is proved.
\end{abstract}

\section{Introduction}

We develop the fundamental theory of existence, uniqueness and continuous differentiability of solutions in the space $L_{p}$ of neutral functional differential equations ( $\mathrm{NFDE}$ )

$$
\text { (1.1) } \frac{d}{d t} E(x(t), x(t-r))=f(x(t), x(t-r))+\int_{-r}^{0} a(\theta) g(x(t+\theta)) d \theta \text {, }
$$

with appropriate continuously Fréchet-differentiable maps $E: X \times X \rightarrow$ $X, f: X \times X \rightarrow X$ and $g: X \rightarrow X$, and an integrable function $a$ from $[-r, 0]$ into the space $\mathcal{L}(X)$ of linear continuous operators on $X, X=\mathbb{R}^{n}$ or $\mathbf{C}^{n}$, with a positive constant delay $r$. For $1 \leq p \leq \infty$, we write $L_{p}$ to denote the Lebesgue space $L_{p}([-r, 0], X)$ and write the above equation, as usual, as

$$
\frac{d}{d t} \mathcal{D}\left(x_{t}\right)=F\left(x_{t}\right)
$$

where the functionals $\mathcal{D}$ and $F$ are defined on the space $C$ of continuous functions $\varphi:[-r, 0] \rightarrow X$ by

$$
\begin{aligned}
& \mathcal{D}(\varphi)=E(\varphi(0), \varphi(-r)) \\
& F(\varphi)=f(\varphi(0), \varphi(-r))+\int_{-r}^{0} a(\theta) g(\varphi(\theta)) d \theta
\end{aligned}
$$


and $x_{t}(\theta) \equiv x(t+\theta), t$ in $(-\infty, \infty), \theta$ in $[-r, 0]$.

The flow of equation (1.1) is the group $\{\mathcal{S}(t)\}_{t \in \mathbb{R}}$ of stronglycontinuous Hadamard-differentiable operators $\mathcal{S}(t)$ on $X \times L_{p}$ defined by

$$
\mathcal{S}(t)(\xi, \varphi)=\left(\xi+\int_{0}^{t} F\left(x_{s}\right) d s, x_{t}\right)
$$

for any $\xi \in X, \varphi \in L_{p}$, where $x \in L_{p}^{\mathrm{loc}}(\mathbb{R}, X)$ is the solution of the integral equation

$$
\mathcal{D}\left(x_{t}\right)=\xi+\int_{0}^{t} F\left(x_{s}\right) d s
$$

which satisfies the initial condition $x_{0}=\varphi$.

We prove that if $1 \leq p<\infty$, then $(S(t))_{t \in \mathbb{B}}$ is a strongly-continuous group and, unless $\mathcal{D}$ is linear, Fréchet differentiability of each map $S(t), t \neq 0$, can not be obtained by Contraction Principles.

We also extend the theorem of Hartman-Grobman on the conjugacy near a hyperbolic equilibrium point of the flow $(S(t))_{t \in \mathbb{E}}$ and the flow of the linearized equation.

We observe that the results can be extended to more general equations such as

$$
\begin{aligned}
& \frac{d}{d t} E\left(x(t), x\left(t-r_{1}\right), \ldots, x\left(t-r_{N}\right), \int_{-r}^{0} a_{1}(\theta) g_{1}(x(t+\theta)) d \theta\right) \\
& \quad=f\left(x(t), x\left(t-r_{1}\right), \ldots, x\left(t-r_{N}\right), \int_{-r}^{0} a(\theta) g(x(t+\theta)) d \theta\right)
\end{aligned}
$$

where $E$ and $f$ are smooth maps from $X^{N+2}$ into $X, g_{1}$ and $g$ are smooth maps from $X$ into itself, $a_{1}$ and $a$ are piecewise continuously differentiable maps from $[-r, 0]$ into $\mathcal{L}(X), 0<r_{1}<\cdots<r_{N}=r$.

We note also that the space $X$ in Theorems 3.1, 3.2, 5.1 (i)-(iv) and 5.3 can be any Banach space and, in Theorem 5.5, any Hilbert space.

\section{Differentiable functions and Contraction Principles}

The results of this section are taken from [9].

Let $E$ and $F$ be complex Banach spaces, $A \subset E$ an open set, $q \in A$ and $f: A \rightarrow F$.

We say that $f$ is Gateaux-differentiable at $q$ if there exists a map $\delta f(q, \cdot): E \rightarrow F$ such that for each $h \in E$ we can write $f(q+t h)=f(q)+$ $t \delta f(q, h)+t R(t, h)$ where $R(t, h) \rightarrow 0$ as $t \rightarrow 0$ in $\mathbb{R}$. The $G$-derivative 
$\delta f(q$,$) is uniquely defince and satisfies the identity \delta f(q, t h)=t \delta f(q, h)$ for any $(t, h)$ in $\mathbb{R} \times E$.

We say that $f$ is Hadamard-differentiable (or quasi-differentiable) at $q$ if there exists a linear map $f^{\prime}(q): E \rightarrow F$ such that for each $h \in E$ we can write $f(q+t h+t k)=f(q)+t f^{\prime}(q) h+R(t, k, h)$ where $R(t, k, h) \rightarrow 0$ as $(t, k) \rightarrow(0,0)$ in $\mathbb{R} \times E$. The $H$-derivative $f^{\prime}(q)$ is also uniquely defined. Clearly we have $\delta f(q, \cdot)=f^{\prime}(q)$ whenever this last exists.

We note that if $\operatorname{dim} E<\infty$, then $f$ is $H$-differentiable if and only if $f$ is Fréchet-differentiable.

Recall that the Lipschitz constant of a map $f$ is defined by

$$
\operatorname{Lip} f=\inf \{L:\|f(q)-f(\bar{q})\| \leq L\|q-\bar{q}\|, q, \bar{q} \in A\} .
$$

Theorem 2.1. If $f$ is $G$-differentiable at $q, \delta f(q, \cdot)$ is linear and there exists a neighborhood $V$ of the point $q$ such that $\operatorname{Lip}\left(f_{\mid V}\right)<\infty$, then $f$ is $H$-differentiable at $q$.

The proof follows easily from the inequalities

$$
\begin{aligned}
& \|f(q+t h+t k)-f(q)-t \delta f(q, h)\| \\
& \quad \leq\|f(q+t h+t k)-f(q+t h)\|+\|f(q+t h)-f(q)-t \delta f(q, h)\|
\end{aligned}
$$

and $\|f(q+t h+t k)-f(q+t h)\| \leq \operatorname{Lip}\left(f_{\mid V}\right)\|t k\|$.

The chain-rule can also be easily proved: the map $f \circ g$ is $H$ differentiable at $q$ if $g$ is $H$-differentiable at $q$ and $f$ is $H$-differentiable at $g(q)$ and $(f \circ g)^{\prime}(q)=f^{\prime}(g(q)) g^{\prime}(q)$.

Theorem 2.2. Let $E$ and $F$ be Banach spaces, $A \subset E \times F$ be an open set and $f: A \rightarrow F$ be a map satisfying the following assumptions:

(i) $f$ has strongly continuous partial $H$-derivatives $f_{x}$ and $f_{y}$ at any point $(x, y)$ in $A$, that is, the maps $(x, y, h) \mapsto f_{x}(x, y) h$ and $(x, y, k) \mapsto f_{y}(x, y) k$ are continuous on $A \times E$ and $A \times F$ respectively.

(ii) There exist closed sets $S$ and $T, S \subset E, T \subset F$ such that $S \times T \subset$ $A, f(S \times T) \subset T$ and $\sup _{(x, y) \in S \times T}\left\|f_{y}(x, y)\right\|<1$.

Then, there is a unique map $y: S \rightarrow T$ such that $y(x)=f(x, y(x))$ for each $x$ in $S$.

Moreover, $y$ is strongly-continuous $H$-differentiable and $y^{\prime}(x)=\{I-$ $\left.f_{y}(x, y(x))\right]^{-1} f_{x}(x, y(x))$ for each $x$ in $S$.

Proof: For each $x$ in $S$, we use the Contraction Mapping Prirnciple to the map $y \in T \mapsto f(x, y) \in T$ to find the fixed point $y(x)$. 
Now, let $\Delta=y(x+t h+t k)-y(x)-t L h$, for small $(t, k) \in \mathbb{R} \times E$, with $L=\left[I-f_{y}(x, y(x))\right]^{-1} f_{x}(x, y(x)), x$ in $S$. Let us call $y=y(x)$. Since

$$
\begin{aligned}
\Delta= & f(x+t h+t k, y+t L h+\Delta)-f(x+t h+t k, y+t L h) \\
& +f(x+t h+t k, y+t L h)-f(x, y)-f_{x}(x, y) t h-f_{y}(x, y) t L h,
\end{aligned}
$$

it follows that

$$
|\Delta| \leq \int_{0}^{l}\left|f_{y}(x+t h+t k, y+t L h+\xi \Delta) \Delta\right| d \xi+o(t) \leq \theta|\Delta|+o(t),
$$

where $\theta=\sup _{(x, y) \in S \times T}\left\|f_{y}(x, y)\right\|<1$ and therefore, $y$ is $H$ differentiable at $x$. The strong-continuity of $y^{\prime}$ follows from the strongcontinuity of $f_{x}$ and $f_{y}$, and the proof is complete.

Theorem 2.3. Let $I \subset \mathbb{R}$ be a compact interval and $X, Y$ be Banach spaces. Let $f: I \times X \rightarrow Y$ be a map satisfying the following conditions

(i) For alnost all $t \in I$, the map $x \mapsto f(t, x)$ is $H$-differentiable and there exists a constant $M>0$ such that $\left\|\frac{\partial f}{\partial x}(t, x)\right\|_{\mathcal{L}(X, Y)} \leq M$, almost everywhere (a.e.) for $t$ in $I$, for all $x$ in $X$.

(ii) For each $x$ and $\bar{x}$ in $X$, the maps $t \mapsto f(t, x)$ and $t \mapsto \frac{\partial f}{\partial x}(t, x) \bar{x}$ are measurable and the map $t \mapsto f(t, 0)$ is in $L_{p}(I, Y)$, for some $p \in[1, \infty)$.

For a given $u \in L_{p}(I, X)$ we define $\tilde{f}(u): I \rightarrow Y$ by $\tilde{f}(u)(t)=$ $f(t, u(t))$ a.e. for $t$ in $I$. Then, $\tilde{f}(u)$ is in $L_{p}(I, Y)$ and the map $\tilde{f}$ : $L_{p}(I, X) \rightarrow L_{p}(I, Y)$ is Lipschitz strongly-continuous $H$-differentiable with $\left[\tilde{f}^{\prime}(u) v\right](t)=\frac{\partial f}{\partial t}(t, u(t)) v(t)$ a.e. for $t$ in $I$, for all $u$ and $v$ in $L_{p}(I, X)$.

Moreover, if $\tilde{f}$ is Fréchet-differentiable at the null function of $L_{p}(I, X)$, then

$$
f(t, x)=f(t, 0)+\frac{\partial f}{\partial x}(t, 0) x
$$

a.e. for $t$ in $I$, for all $x \in X$.

Proof: For the proof that $\widetilde{f}(u)$ is in $L_{p}(I, Y)$ see [11]. First, we prove that $\tilde{f}$ is $G$-differentiable. We have

$$
\begin{aligned}
& \frac{1}{s}[\tilde{f}(u+s v)(t)-\tilde{f}(u)(t)]-\frac{\partial f}{\partial x}(t, u(t)) v(t) \\
& =\frac{1}{s} \int_{0}^{s}\left[\frac{\partial f}{\partial x}(t, u(t)+\sigma v(t))-\frac{\partial f}{\partial x}(t, u(t))\right] v(t) d \sigma-\rightarrow 0
\end{aligned}
$$


a.e. for $t$ in $I$. On the other hand, for any measurable $E \subset I$ we have

$$
\begin{aligned}
\left\|\frac{1}{s}[\tilde{f}(u+s v)-\tilde{f}(u)]-\frac{\partial f}{\partial x}(\cdot, u(\cdot)) v(\cdot)\right\|_{L_{p}(E, Y)} \\
\quad \leq\left|\frac{1}{2} \int_{0}^{s} 2 M\|v\|_{L_{p}(E, X)} d \sigma\right|=2 M\|v\|_{L_{p}(E, X)} \longrightarrow 0
\end{aligned}
$$

uniformly in $0<|s|<$ constant, when the measure of $E$ tends to zero. This imply that

$$
\left\|\frac{1}{s}[\tilde{f}(u+s v)-\tilde{f}(u)]-\frac{\partial f}{\partial x}(\cdot, u(\cdot)) v(\cdot)\right\|_{p} \longrightarrow 0
$$

when $s \rightarrow 0$, and therefore the $G$-derivative of $\widetilde{f}$ exists and $\delta \tilde{f}(u, v)(t)=$ $\frac{\partial f}{\partial x}(t, u(t)) v(t)$ a.e. for $t$ in $I$ and it is linear on $v$.

Since

$$
\begin{aligned}
\|\tilde{f}(u)-\tilde{f}(v)\|_{p}^{p} & \leq \int_{I}\left[\int_{0}^{1}\left|\frac{\partial f}{\partial x}(t, \xi u(t)+(1-\xi) v(t))(u(t)-v(t))\right| d \xi\right]^{p} d t \\
& \leq \int_{I} M^{p}|u(t)-v(t)|^{p} d t=M^{p}\|u-v\|_{p}^{p}
\end{aligned}
$$

it follows that $\tilde{f}$ is Lipschitz continuous. By Theorem 2.1, $\tilde{f}$ is $H$ differentiable.

Now, $\delta \widetilde{f}(u, v)$ is continuous since $u_{n} \rightarrow u$ and $v_{n} \rightarrow v$ in $L_{p}(I, X)$ imply $\delta \tilde{f}\left(u_{n}, v_{n}\right) \rightarrow \delta \tilde{f}(u, v)$ in measure, and $\int_{E}\left|\delta \tilde{f}\left(u_{n}, v_{n}\right)(t)\right|^{p} d t \leq$ $M^{p} \int_{E}\left|v_{n}(t)\right|^{p} d t \rightarrow 0$, uniformly in $n$, when the measure of $E$ tends to zero. This imply that $\delta \tilde{f}\left(u_{n}, v_{n}\right) \rightarrow \delta \tilde{f}(u, v)$ in $L_{p}(I, X)$.

To prove the last assertion, we can assume without loss of generality that $f(t, 0) \equiv 0$. If there exists $x_{0} \neq 0$ such that $f\left(t, x_{0}\right)-\frac{\partial f}{\partial x}(t, 0) x_{0} \equiv$ $\Delta(t)$ with $\Delta(t) \neq 0$ in a set of positive measure, then we can take a point $t_{0}$ in the interior of $I$ such that $\lim _{\delta \rightarrow 0^{+}} \frac{1}{2 \delta} \int_{t_{0}-\delta}^{t_{0}+\delta}|\Delta(t)|^{p} d t=\left|\Delta\left(t_{0}\right)\right|^{p} \neq 0$.

Taking the function $v_{\delta}(t)=x_{0}$ for $t \in\left[t_{0}-\delta, t_{0}+\delta\right]$ and $v_{\delta}(t) \equiv 0$ outside, we have $\left\|v_{\delta}\right\|_{p}=\left|x_{0}\right|(2 \delta)^{1 / p} \rightarrow 0$ when $\delta \rightarrow 0^{+}$, but

$$
\begin{aligned}
& \frac{\left\|\tilde{f}\left(v_{\delta}\right)-\tilde{f}(0)-\tilde{f}^{\prime}(0) v_{\delta}\right\|_{p}^{p}}{\left\|v_{\delta}\right\|_{p}^{p}} \\
& \quad=\frac{\int_{t_{0}-\delta}^{t_{0}+\delta}\left|f\left(t, x_{0}\right)-\frac{\partial f}{\partial x}(t, 0) x_{0}\right|^{p} d t}{\left|x_{0}\right|^{p} 2 \delta} \longrightarrow \frac{\left|\Delta\left(t_{0}\right)\right|^{p}}{\left|x_{0}\right|^{p}} \neq 0,
\end{aligned}
$$

a contradiction with the hypothesis that $\widetilde{f}$ is Fréchet-differentiable at 0 . Therefore, $f(t, x)=\frac{\partial f}{\partial x}(t, 0) x$ a.e. for $t$ in $I$, for all $x$ in $X$, and the proof is complete. 


\section{The fundamental theory}

We state the fundamental theorems on the solutions of the NFDE

$$
\frac{d}{d t} E(x(t), x(t-r))=f(x(t), x(t-r))+\int_{-r}^{0} a(\theta) g(x(t+\theta)) d \theta
$$

where $E$ and $f$ are continuously Fréchet-diferentiable maps from $X \times X$ into $X, X$ is the $n$-dimensional Euclidean space $\mathbb{R}^{n}$ or $C^{n}, g: X \rightarrow X$ is also continuously Fréchet-differentiable and $a$ is an integrable map from $[-r, 0]$ into the space $\mathcal{L}(X)$ of continuous lincar operators from $X$ into itself.

We fix $p, 1 \leq p \leq \infty$, and look for solutions of $(3.1)$ in $L_{p}^{\text {loc }}(\mathbb{R}, X)$.

We assume that:

(H1) The derivatives $D E \equiv\left[\frac{\partial E}{\partial p_{0}} \frac{\partial E}{\partial p_{r}}\right], D f \equiv\left[\frac{\partial f}{\partial p_{0}} \frac{\partial f}{\partial p_{r}}\right]$ and $D g$ are all bounded on their domains.

(H2) There exist injective continuous linear operators $L_{0}$ and $L_{r}$ from $X$ into itself and a real constant $c, 0 \leq c<1$, such that

$$
\left\|I-L_{0} \frac{\partial E}{\partial p_{0}}\left(p_{0}, p_{r}\right)\right\| \leq c \text { and }\left\|I-L_{r} \frac{\partial E}{\partial p_{r}}\left(p_{0}, p_{r}\right)\right\| \leq c
$$

for all $\left(p_{0}, p_{r}\right) \in X \times X$, where $I$ is the identity.

(H3) $\|a\|_{p^{\prime}}<\infty$, where $\frac{1}{p}+\frac{1}{p^{\prime}}=1$.

We observe that if $x$ belongs to $L_{p}^{\text {loc }}(\mathbb{R}, X)$, then the maps $t \mapsto \mathcal{D}\left(x_{t}\right)=$ $E(x(t), x(t-r))$ and $t \mapsto F\left(x_{t}\right)=f(x(t), x(t-r))+\int_{-r}^{0} \alpha(\theta) g(x(t+\theta)) d \theta$ also belong to $L_{p}^{\text {loc }}(\mathbb{R}, X)$.

We say that $x$ in $L_{p}^{\mathrm{loc}}(\mathbb{R}, X)$ is a solution of (3.1) if there exists a constant vector $\xi$ in $X$ such that the relation

$$
\mathcal{D}\left(x_{t}\right)=\xi+\int_{0}^{t} F\left(x_{s}\right) d s
$$

holds a.e. for $t$ in $(-\infty, \infty)$; in this case, $x$ is also a solution of the integral equation (3.2).

We denote by $L_{p}$ the Banach spacc $L_{p}([-r, 0], X)$ with the $p$-norm.

Theorem 3.1. For each $(\xi, \varphi) \in X \times L_{p}$ there exists a unique solution $x$ in $L_{p}^{\text {loc }}(\mathbb{R}, X)$ of equation (3.2) such that $x_{0}=\varphi$. Moreover, if $1 \leq p<\infty$, the map $(t, \xi, \varphi) \mapsto x_{t} \in L_{p}$ is continuous. This map is 
not continuous when $p=\infty$ although for each $t$ in $(-\infty, \infty)$, the map $(\xi, \varphi) \mapsto x_{t} \in L_{\mathrm{co}}$ is continuous.

Proof: Given $(\xi, \varphi) \in X \times L_{p}$, we consider the map $\mathcal{F}$ from $L_{p}([0, T], X)$ into itself defined by

$$
\mathcal{F}[y](t)=y(t)-L_{0}\left[\mathcal{D}\left(y_{t}\right)-\xi-\int_{0}^{t} F\left(y_{s}\right) d s\right] \text { a.e. for } t \text { in }[0, T]
$$

where we put $y_{0}=\varphi$.

For sufficiently small $T>0$, independent of $(\xi, \varphi), \mathcal{F}$ is a contraction, uniformly in $(\xi, \varphi)$. The fixed point $x$ of $\mathcal{F}$ is the solution defined on $[0, T]$.

We use an induction argument to obtain the solution on $[0, \infty)$.

Analogously, the solution on $[-T-r,-r]$ is the fixed point of the map $\widetilde{\mathcal{F}}$ defined by

$\tilde{\mathcal{F}}[y](t-r)=y(t-r)-L_{r}\left[\mathcal{D}\left(y_{t}\right)-\xi-\int_{0}^{t} F\left(y_{s}\right) d s\right]$ a.e. for $t$ in $[-T, 0]$,

where again we put $y_{0}=\varphi$.

Again, by induction, we get the solution on $(-\infty, \infty)$.

Since the map $t \mapsto x_{t} \in L_{p}$ is continuous when $1 \leq p<\infty$ and not continuous when $p=\infty$, the rest of the proof is a consequence of the continuity of each contraction with respect to $(\xi, \varphi, y)$ and the independence on $(\xi, \varphi)$ of the contraction constant.

The flow corresponding to equation (3.2) is the family $\{S(t)\}_{t \in \mathbb{B}}$ of operators $\mathcal{S}(t)$ from $X \times L_{p}$ onto itself defined by

$$
\mathcal{S}(t)(\xi, \varphi)=\left(\xi+\int_{0}^{t} F\left(x_{s}\right) d s, x_{\imath}\right)
$$

where $x=x(\xi, \varphi)$ is the solution of equation (3.2) through $(\xi, \varphi)$.

By Theorem 3.1, the flow is a group of homeomorphisms under composition which is strongly continuous when $1 \leq p<\infty$.

Theorem 3.2. Suppose that $E, f$ and $g$ are turce continuously Fréchet-differentiable with

$$
\left\|D^{(2)} E\right\|,\left\|D^{(2)} f\right\| \text { and }\left\|D^{(2)} g\right\|
$$


bounded on their domains. The following statements are true:

(i) For any $t$ in $(-\infty, \infty), \mathcal{S}(t)$ is strongly-continuous $H$-differentiable at any point $(\xi, \varphi)$ and $D S(t)(\xi, \varphi)(\Delta \xi, \Delta \varphi)=(\Delta \xi+$ $\left.\int_{0}^{t} M(s) z_{s} d s, z_{t}\right)$ where $z$ is the solution of the linearized equation around the solution $S(t)(\xi, \varphi)$

$$
\begin{aligned}
A_{0}(t) z(t)+A_{r}(t) z(t-r) & \\
= & \Delta \xi+\int_{0}^{t} M(s) z_{s} d s \text { a.e for } t \text { in }(-\infty, \infty)
\end{aligned}
$$

through $(\Delta \xi, \Delta \varphi)$, where $M(t) z_{t}=B_{0}(t) z(t)+B_{r}(t) z(t-$ $r)+\int_{-r}^{0} B(t, \theta) z(t+\theta) d \theta, B(t, \theta)=a(\theta) D g(x(t+\theta)), B_{i}(t)=$ $\frac{\partial f}{\partial p_{i}}(x(t), x(t-r))$ and $A_{\imath}(t)=\frac{\partial E}{\partial p_{i}}(x(t), x(t-r))$ a.e. for $t$ in $(-\infty, \infty), i=0, r$

(ii) Suppose $2 \leq p \leq \infty$ and $a$ is essentially bounded. If $E$ is linear then $S(t)$ is continuously $F$-differentiable (class $C^{1}$ ) for any $t$ in $\mathbb{R}$. Conversely, when $p$ is not $\infty$, if either the operator $\mathcal{F}$ or the operator $\widetilde{\mathcal{F}}$ given in the proof of Theorem 3.1 is Fréchetdifferentiable at some point $(\xi, \varphi, y)$ in its domain, then $E$ is linear.

Proof: (i) The proof follows from Theorem 2.2 applied to the contraction $\mathcal{F}$ given by relation (3.3) above, as follows: $\mathcal{F}$ is $G$-differentiable with respect to $(\xi, \varphi, y)$ in $X \times L_{p} \times L_{p}([0, T], X)$, the derivative at $(\xi, \varphi, y)$ is the continuous linear map given by

$$
\begin{aligned}
D \mathcal{F} & (\xi, \varphi, y)(\Delta \xi, \Delta \varphi, \Delta y)(t) \\
& =\Delta y(t)-L_{0}\left[A_{0}(t) y(t)+A_{r}(t) y(t-r)-\Delta \xi-\int_{0}^{t} M(s) y_{s} d s\right]
\end{aligned}
$$

a.e. for $t$ in $[0, T]$, and $\mathcal{F}$ is Lipschitizian. Therefore, by Theorem $2.1, \mathcal{F}$ is $H$-differentiable.

To prove that $D \mathcal{F}$ is strongly-continuous we first prove the result when $\Delta \varphi$ and $\Delta y$ are essentially bounded and use a density argument for the general case.

Now, for $T>0$ sufficiently small, it is easy to see that $\sup \left\{\left\|\frac{\partial \mathcal{F}}{\partial y}(\xi, \varphi, y)\right\|: \xi\right.$ in $X, \varphi$ in $L_{p}, y$ in $\left.L_{p}([0, T], X)\right\}$ is less than 1 . By Theorem 2.2, the fixed point $x(\xi, \varphi)$ is strongly-continuous $H$ differentiable.

Since, for $0 \leq t \leq T$, the $\operatorname{map}(\xi, \varphi) \mapsto(\xi, x(\xi, \varphi))$ and $(\xi, y) \mapsto$ $\left(\xi+\int_{0}^{t} F\left(y_{s}\right) d s, y_{t}\right)$ are strongly-continuous $I I$-differentiable, so is the composition $\mathcal{S}(t)$. 
For an arbitrary positive $t$, we take a positive integer $m$ such that $0<\frac{t}{m} \leq T$. Since $S(t)=S(t / m)^{m}$, the chain-rule shows that $S(t)$ is strongly-continuous $H$-differentiable.

The proof for negative $t$ is the same if we use the contraction $\widetilde{\mathcal{F}}$.

The strongly-continuous $H$-differentiability of $(\xi, \varphi) \mapsto x(\xi, \varphi) \in$ $L_{p}([a, b], X)$ for an arbitrary compact interval $[a, b]$ and the proof that $z=D x(\xi, \varphi)(\Delta \xi: \Delta \varphi)$ satisfies equation (3.4) follow easily from the differentiability of $\mathcal{S}(t)$ and the chain-rule applied to the equation (3.2).

(ii) Let us show that $\mathcal{F}$ is of class $C^{1}$. Since $E$ is linear, it suffices to show that $(\varphi, y) \mapsto \mathcal{F}^{*}(\varphi, y)$ is $C^{1}$, where

$$
\mathcal{F}^{*}(\varphi, y)(t)=\left\{\begin{aligned}
\int_{0}^{t}[f(y(s), \varphi(s-r)) & +\int_{-r}^{-s} a(\theta) g(\varphi(s+\theta)) d \theta \\
& \left.+\int_{-s}^{0} a(\theta) g(y(s+\theta)) d \theta\right] d s \\
& \text { if } 0 \leq t \leq r \\
& \text { if } T>r \text { and } r \leq t \leq T .
\end{aligned}\right.
$$

We show that $\mathcal{F}^{*}$ is of class $C^{1}$ with respect to $\varphi$.

Using the $H$-derivative of $\mathcal{F}^{*}$, taken from (i), we have

$$
\begin{aligned}
& \mid \mathcal{F}^{*}(\varphi+\Delta \varphi, y)(t)-\mathcal{F}^{*}(\varphi, y)(t)-\frac{\partial \mathcal{F}^{*}}{\partial \varphi}(\varphi, y) \Delta \varphi(t) \mid \\
& \leq \int_{0}^{t} \frac{1}{2}\left\|D^{(2)} f\right\||\Delta \varphi(s-r)|^{2} d s \\
&+\int_{0}^{t} \frac{1}{2}\left\|D^{(2)} g\right\| \int_{-r}^{-s}|a(\theta) \| \Delta \varphi(s+\theta)|^{2} d \theta d s \\
& \leq \frac{1}{2}\left\|D^{(2)} f\right\| t^{1 / \widetilde{p}}\|\Delta \varphi\|_{p}^{2}+\frac{1}{2}\left\|D^{(2)} g\right\|\|a\|_{p}\|\Delta \varphi\|_{p}^{2} t
\end{aligned}
$$

a.e. for $t$ in $[0, T]$, where $\frac{1}{\tilde{p}}+\frac{2}{p}=1$. It follows that

$$
\left\|\mathcal{F}^{*}(\varphi+\Delta \varphi, y)-\mathcal{F}^{*}(\varphi, y)-\frac{\partial \mathcal{F}^{*}}{\partial \varphi}(\varphi, y) \Delta \varphi\right\|_{p} \leq K T^{1 / p}\|\Delta \varphi\|_{p 1}^{2}
$$

where $K$ is constant. In the same way it may be shown that $\mathcal{F}^{*}$ is $C^{1}$ with respect to $y$. To prove the continuity of $\frac{\partial \mathcal{F}^{*}}{\partial \varphi}: L_{p} \times L_{p}([0, T], X) \rightarrow$ $\mathcal{L}\left(L_{p}, L_{p}([0, T], X)\right)$ with respect to $\varphi$, for example, we have

$$
\begin{aligned}
&\left|\left(\frac{\partial \mathcal{F}^{*}}{\partial \varphi}(\varphi, y)-\frac{\partial \mathcal{F}^{*}}{\partial \varphi}(\psi, y)\right) \Delta \varphi(t)\right| \leq\left\|D^{(2)} f\right\|\|\varphi-\psi\|_{p}\|\Delta \varphi\|_{p^{\prime}} \\
&+\left\|D^{(2)} g\right\|\|a\|_{\infty}\|\varphi-\psi\|_{p}\|\Delta \varphi\|_{p^{\prime}} r \\
& \leq\left(\left\|D^{(2)} f\right\| r^{\frac{p-2}{p}}\right. \\
&+\left\|D^{(2)} g\right\|\|a\|_{\infty} r^{\frac{2 p-2}{p}}\|\| \Delta \varphi\left\|_{p}\right\| \varphi-\psi \|_{p} \\
&=\bar{K}\|\Delta \varphi\|_{p}\|\varphi-\psi\|_{p}
\end{aligned}
$$


a.e. for $t$ in $[0, T]$, where $\bar{K}$ is a constant. Note that $p^{\prime} \leq p$. It follows that

$$
\left\|\frac{\partial \mathcal{F}^{*}}{\partial \varphi}(\varphi, y) \Delta \varphi-\frac{\partial \mathcal{F}^{*}}{\partial \varphi}(\psi, y) \Delta \varphi\right\|_{p} \leq \bar{K} T^{1 / p}\|\Delta \varphi\|_{p}\|\varphi-\psi\|_{p}
$$

and therefore

$$
\left\|\frac{\partial \mathcal{F}^{*}}{\partial \varphi}(\varphi, y)-\frac{\partial \mathcal{F}^{*}}{\partial \varphi}(\psi, y)\right\|_{\mathcal{L}\left(L_{p}, L_{p}([0, T], X)\right)} \leq \bar{K} T^{1 / p}\|\varphi-\psi\|_{p} .
$$

When $T>0$ is small enough, the fixed point $x(\xi, \varphi)$ of $\mathcal{F}(\xi, \varphi, \cdot)$ is of class $C^{1}$ in $(\xi, \varphi)$. The proof that $S(t)$ is of class $C^{1}$ for an arbitrary $t$ in $(-\infty, \infty)$ follows as in item (i), changing "strongly-continuous $H$ differentiable" for "continuously Fréchet-differentiable".

Suppose now that $\mathcal{F}$ is Fréchet-differentiable at some point $\left(\xi_{0}, \varphi_{0}, y_{0}\right)$ (the case of $\widetilde{\mathcal{F}}$ is completely analogous). Define $\widetilde{E}: L_{p} \times L_{p}([0, T], X) \rightarrow$ $L_{p}([0, T], X)$ by $\widetilde{E}(\varphi, y)(t)=E(y(t), y(t-r))$ a.e. for $t$ in $[0, T]$, where we put $y(\theta)=\varphi(\theta)$ for $\theta$ in $[-r, 0]$. Then we have $\mathcal{F}(\xi, \varphi, y)=y-$ $L_{0} \tilde{E}(\varphi, y)+L_{0}(\xi)+L_{0} \mathcal{F}^{*}(\varphi, y)$. This implies that $\widetilde{E}$ is $F$-differentiable at $\left(\varphi_{0}, y_{0}\right)$, since the other parcels are of class $C^{1}$ in $(\xi, \varphi, y)$. There is no loss of generality in supposing that $0<T \leq r$.

We now use the Theorem 2.3. Let $I=[0, T]$ and use in $X^{2}=X \times X$ the norm $\left\|\left(p_{0}, p_{r}\right)\right\|=\left(\left|p_{0}\right|^{p}+\left|p_{r}\right|^{p}\right)^{1 / p}$.

Define $f: I \times X^{2} \rightarrow X$ by $f\left(t,\left(p_{0}, p_{r}\right)\right)=E\left(y_{0}(t)+p_{0}, \varphi_{0}(t-r)+\right.$ $\left.p_{r}\right)-E\left(y_{0}(t), \varphi_{0}(t-r)\right)$ a.e. for $t$ in $I$, for all $\left(p_{0}, p_{r}\right)$ in $X^{2}$. For a given $u \in L_{p}\left(I, X^{2}\right), u(t)=\left(u_{1}(t), u_{2}(t)\right)$, we have $\tilde{f}(u)(t) \stackrel{\text { a.e. }}{=} E\left(y_{0}(t)+\right.$ $\left.u_{1}(t), \varphi_{0}(t-r)+u_{2}(t)\right)-E\left(y_{0}(t), \varphi_{0}(t-r)\right)$. We have $f(t, 0) \equiv 0$ and $\vec{f}$ is $F^{\prime}$-differentiable at the null function of $L_{p}\left(I, X^{2}\right)$. Then, by Theorem 2.3, $f\left(t,\left(p_{0}, p_{r}\right)\right) \stackrel{\text { a.e. }}{=} \frac{\partial f}{\partial p_{0}}(t,(0,0)) p_{0}+\frac{\partial f}{\partial p_{r}}(t,(0,0)) p_{\tau}$, that is,

$$
\begin{aligned}
& E\left(y_{0}(t)+p_{0}, \varphi_{0}(t-r)+p_{r}\right) \\
= & E\left(y_{0}(t), \varphi_{0}(t-r)\right)+\frac{\partial E}{\partial p_{0}}\left(y_{0}(t), \varphi_{0}(t-r)\right) p_{0}+\frac{\partial E}{\partial p_{r}}\left(y_{0}(t), \varphi_{0}(t-r)\right) p_{r}
\end{aligned}
$$

a.e. for $t$ in $I$, for all $\left(p_{0}, p_{r}\right)$ in $X^{2}$. If we take some dense and countable set $\mathcal{D} \subset X^{2}$, we can find some $t \in I$ for which the above equality holds at this $t$ and all $\left(p_{0}, p_{r}\right) \in \mathcal{D}$. By the continuity of $E$ we get

$$
E\left(p_{0}, p_{r}\right)=E(0,0)+\frac{\partial E}{\partial p_{0}}(0,0) p_{0}+\frac{\partial E}{\partial p_{r}}(0,0) p_{r}
$$

for all $\left(p_{0}, p_{r}\right)$ in $X^{2}$, and the proof is complete. 


\section{The linear flow}

If $E, f$ and $g$ are linear, the equation (3.2) can be written as

$$
D y_{t}=\xi+\int_{0}^{t} L y_{s} d s \text { a.e. for } t \text { in }(-\infty, \infty)
$$

where

$$
\begin{aligned}
& D y_{t}=A_{0} y(t)+A_{r} y(t-r), \\
& L y_{t}=B_{0} y(t)+B_{r} y(t-r)+\int_{-r}^{0} B(\theta) y(s+\theta) d \theta,
\end{aligned}
$$

a.e. for $t \in(-\infty, \infty)$. The functions $A_{0}, A_{r}, B_{0}, B_{r}$ are in $\mathcal{L}(X), B$ is in $L_{p^{\prime}}([-r, 0], \mathcal{L}(X))$ and the hypothesis (H2) bccomes: $A_{0}$ and $A_{r}$ are invertible in $\mathcal{L}(X)$. In this case, the flow of cquation (4.1), denoted by $\{T(t)\}_{t \in \mathbb{R}}$, is a group of linear isomorphisms on $X \times L_{p}$.

We will need also the non-homogeneous equation

$$
D y_{t}=\xi+\int_{t_{0}}^{t} L y_{s} d s+Q(t) \text { a.e. for } t \text { in }(-\infty, \infty)
$$

where $Q$ is in $L_{p}^{\text {loc }}(\mathbb{R}, X), t_{0} \in \mathbb{R}$.

The existence and uniqueness of solution $y$ in $L_{p}^{\operatorname{loc}}(\mathbb{R}, X)$ for equation (4.2) with initial conditions $y_{t_{0}}=\varphi$ can be proved with the same arguments used in Theorem 3.1. If $1 \leq p<\infty$, the solution depends continuously on $\xi$ in $X, \varphi$ in $L_{p}, t_{0}$ in $\mathbb{R}$ and $Q_{|| a, b]}$ in $L_{p}([a, b], X)$, for each compact interval $[a, b]$ containing $t_{0}$, and the solution $y\left(t_{0}, \xi, \varphi, Q\right)$ can be decomposed as $y_{t}\left(t_{0}, \xi, \varphi, Q\right)=\pi_{2} T\left(t-t_{0}\right)(\xi, \varphi)+K\left(t, t_{0}\right) Q_{\mid\left[t_{0}, t\right]}$, where $\pi_{2}$ is the projection $(\xi, \varphi) \mapsto \varphi$ and $K\left(t, t_{0}\right)$ is a continuous linear operator from $L_{p}\left(\left[t_{0}, t\right], X\right)$ into $L_{p}$ (if $t<t_{0}$ we interchange the order in $\left.\left[t_{0}, t\right]\right)$.

Let $\left\{T^{0}(t)\right\}_{t \in \mathrm{R}}$ be the flow of the difference equation

$$
D y_{t}=0
$$

The flow $\left\{T^{0}(t)\right\}_{t \in \mathbb{R}}$ is the $\pi_{2}$-projection of the restriction to $\{0\} \times L_{p}$ of the flow corresponding to equation (4.1) with $L \equiv 0$.

The main purpose of this section is to describe the spectra of $T^{0}(t)$ and $T(t)$. Define $H(\lambda) \triangleq A_{0}+A_{r} e^{-\lambda r}$ and $\Delta(\lambda) \triangleq \lambda H(\lambda)-B_{0}-B_{r} e^{-\lambda r}-$ $\int_{-r}^{0} B(\theta) e^{\lambda \theta} d \theta$, for $\lambda$ in $\mathrm{C}$. 
Theorem 4.1. (i) For $1 \leq p<\infty$, the infinitesimal generator of the group $\left\{T^{0}(t)\right\}_{t \in \mathbb{T}}$ is given by $G^{0} \varphi=\dot{\varphi}, \mathcal{D}\left(G^{0}\right)=\left\{\varphi \in W^{1, p}: D(\varphi)=0\right\}$ where $W^{1, p}=\left\{\varphi \in L_{p}: \dot{\varphi} \in L_{p}\right\}$.

(ii) The spectrum of $G^{0}$ consists only on eigenvolues and $\lambda \in \sigma\left(G^{0}\right)$ if and only if $\operatorname{det} H(\lambda)=0$.

(iii) If $p=2$, then $\sigma\left(T^{0}(t)\right)=\overline{e^{t \sigma\left(G^{0}\right\rangle}}$.

Proof: The proof is due to D. Henry [8].

(i) Let $\varphi$ be in $\mathcal{D}\left(G^{0}\right)$. Then, putting $x_{t}=T^{0}(t) \varphi$ and $y_{t}=$ $T^{0}(t) G^{0} \varphi=G^{0} T^{0}(t) \varphi=\frac{d}{d t} T^{0}(t) \varphi=\frac{d}{d t} x_{t}$ we have $x_{t}=\varphi+\int_{0}^{t} y_{s} d s$ so that, a.e. for $\theta$ in $[-r, 0], x(t+\theta)=\varphi(\theta)+\int_{\theta}^{t+\theta} y(s) d s$. Hence, for each $\theta$ in $[-r, 0]$ for which this last relation holds, the right-hand side is an absolutely continuous function of $t$ in $\mathbb{R}$ and so is $x$ with $\dot{x}=y$. Then $\varphi=x_{0}$ is in $W^{1, p}, \dot{\varphi}=y_{0}=G^{0} \varphi$ and $D \varphi=0$ because when the solution is continuous, the equation is satisficd for all $t$ in $\mathbb{R}$.

Conversely, if $\varphi$ is in $W^{1, p}, D \varphi=0$, then, putting $x_{t}=T^{0}(t) \varphi, y_{t}=$ $T^{0}(t) \dot{\varphi}$ and $z(t)=x(t)-\varphi(0)-\int_{0}^{t} y(s) d s$, we have $z_{0}=0$ and $D\left(z_{t}\right)=0$ a.c. for $t$ in $\mathbb{R}$. By uniqueness, $z \equiv 0$, so $x$ is absolutely continuous and $\dot{x}=y$ and then $\varphi$ is in $\mathcal{D}\left(G^{0}\right)$ and $G^{0} \varphi=\dot{\varphi}$.

(ii) For a given $\psi$ in $L_{p}$ and $\lambda$ in $\mathbf{C}$, the solution $\varphi$ of $G^{0} \varphi-\lambda \varphi=\psi$ is given by $\varphi(\theta)=e^{\lambda \theta} \varphi(0)+\int_{-r}^{\theta} e^{\lambda(\theta-u)} \psi(u) d u$. This solution $\varphi$ is in $\mathcal{D}\left(G^{0}\right)$ if and only if $D \varphi=H(\lambda) \varphi(0)+A_{r} \int_{-r}^{0} e^{-\lambda(r+u)} \psi(u) d u$ is equal to zero. This last condition determines $\varphi(0)$ uniquely if and only if $\operatorname{det} H(\lambda) \neq 0$. From this we conclude that $\sigma\left(G^{0}\right)=\{\lambda \in \mathrm{C}: \operatorname{det} H(\lambda)=$ $0\}$ and contains only eigenvalues.

(iii) It is easy to see that, for $\lambda$ such that $\operatorname{Re} \lambda \geq \alpha$ and $\operatorname{det} I(\lambda) \neq 0$, there exists a constant $c_{\alpha}$ such that $\left\|\left(\lambda-G^{0}\right)^{-1}\right\|_{\mathcal{L}\left(I_{2}\right)} \leq$ $c_{\alpha}\left\|H(\lambda)^{-1}\right\|_{\mathcal{L}(X)}$.

A theorem of Gearhart and Herbst [10] says: $e^{\lambda t}$ is in $\sigma\left(T^{0}(t)\right)$ if and only if either there exists an integer $k$ such that $\lambda+\frac{2 \pi i k}{t}$ is in $\sigma\left(G^{0}\right)$ or for any integer $k, \lambda+\frac{2 \pi i k}{t}$ is not in $\sigma\left(G^{0}\right)$ but $\left(\lambda+\frac{2 \pi i k}{t}-G^{0}\right)^{-1}$ is not uniformly bounded as $k \rightarrow \pm \infty$.

If we have this last possibility then $\operatorname{dist}\left(\sigma\left(G^{0}\right),\left\{\lambda+\frac{2 \pi i k}{t}: k\right.\right.$ integer $\})=0$. In fact, $\operatorname{det} H(\lambda)$ is an analytic almost periodic function of $\lambda$, and this implies that $H(\lambda)^{-1}$ is uniformly bounded in any set $\left\{\lambda \in \mathrm{C}: \alpha \leq \operatorname{Re} \lambda \leq \beta, \operatorname{dist}\left(\sigma\left(G^{0}\right), \lambda\right) \geq \delta\right\}$ for $\alpha<\beta, \delta>0$ (see [6]). Therefore, $e^{\lambda t} \in \overline{e^{t \sigma\left(G^{0}\right)}}$ and the proof is complete.

Remark 4.1. We have taken $p=2$ in Theorem 4.1 (iii) because the Theorem of Gearhart and Herbst applies to strongly continuous semi- 
groups defined on Hilbert spaces and no comparable result is known for semigroups on Banach spaces.

Remark 4.2. Theorem 4.1 has been proved in $[8]$ when $X$ is any Hilbert space and $D$ has the special form

$$
D x_{t} \triangleq x(t)+\sum_{k=1}^{\infty} A_{k} x\left(t-r_{k}\right)+\int_{-\tau}^{0} A(\theta) x(t+\theta) d \theta
$$

where $A_{k}$ and $A(\theta)$ are compact operators. The possibility $r=+\infty$ was also allowed.

In the following, we use the identification $T^{0}(t): L_{p} \rightarrow L_{p} \equiv\{0\} \times L_{p}$.

Lemma 4.2. For $1<p \leq \infty$, the map $T(t)-T^{0}(t) \pi_{2}$ is compact.

Proof: The proof follows by observing that $T(t)-T^{0}(t) \pi_{2}=$ $\left(Q, K^{0}(t) Q\right)$, where $K^{0}(t)$ is the above operator $K(t, 0)$ corresponding to equation (4.2) with $L \equiv 0$ and $Q(t)=\pi_{1} T(t)(\xi, \varphi), \pi_{1}$ being the projection $(\xi, \varphi) \mapsto \xi$. The map $(\xi, \varphi) \mapsto Q$ is a compact linear map from $X \times L_{p}$ into the space of continuous functions from $[0, t]$ (or $[t, 0]$ if $\left.t<0\right)$ to $X$ with the uniform norm, by the Arzelá-Ascoli theorem.

Theorem 4.3. (i) For $1 \leq p<\infty$, the infinitesimal generator of the group $\{T(t)\}_{t \in \mathbb{R}}$ is given by $G(\xi, \varphi)=(L \varphi, \dot{\varphi}), \mathcal{D}(G)=\{(\xi, \varphi) \in$ $\left.X \times W^{1, p}: \xi=D \varphi\right\}$.

(ii) The spectrum of $G$ consists only on eigenvalues and $\lambda \in \sigma(G)$ if and only if $\operatorname{det} \Delta(\lambda)=0$.

(iii) If $p=2$, then $\sigma(T(t))=\overline{e^{t \sigma(G)}}$, a.e. for $t$ in $(-\infty, \infty)$.

Proof: The proof of (i) and (ii) follows them same steps of the proof of (i) and (ii) of Theorem 4.1.

(iii) Let $Z=\left\{\operatorname{Re} \lambda: \lambda \in \sigma\left(G^{0}\right)\right\}$, let $(\beta, \gamma)$ be an open bounded interval disjoint from the closure $\bar{Z}$. Let $U=\left\{u \in \mathbf{C}: e^{\beta t}<|u|<e^{\gamma t}\right\}$. Since for all $t$ we have

$$
\overline{e^{t \sigma\left(G^{0}\right)}} \subset\left\{u \in \mathbf{C}:|u|=e^{t \xi}, \xi \in \bar{Z}\right\}
$$

using Theorem 4.1 (iii), we conclude that $U$ is disjoint from $\sigma\left(T^{0}(t)\right.$ ). Also $\sigma\left(T^{0}(t) \pi_{2}\right)=\sigma\left(T^{0}(t)\right) \cup\{0\}$ and $U$ is disjoint from $\sigma\left(T^{0}(t) \pi_{2}\right)$. By Lemma 4.2 and a result of Gohberg and Krein stated in [6, Lemma $4.2]$, it follows that $U \subset \tilde{\rho}(T(t))$ or $U \subset e^{t \sigma(G)}$, where $\tilde{\rho}(T(t))$ is the set of all normal points (regular points or isolated eigenvalues with finite multiplicities) of $T(t)$. 
Since $e^{t \sigma(G)}$ is countabie, $U$ must be disjoint from the essential spectrum $\sigma_{e}(T(t))$, the complement of $\tilde{\rho}(T(t))$, which implies that $\sigma_{e}(T(t))$ is contained in the set $\left\{u \in \mathbf{C}:|u|=e^{t \xi}, \xi \in \bar{Z}\right\}$.

From [7], the inclusion (4.4) is, in fact, an equality holding for almost all $t$ in $(-\infty, \infty)$, so that $\sigma_{e}(T(t))$ is contained in $\overline{e^{t \sigma\left(G^{0}\right)}}$ a.e. for $t$ in $(-\infty, \infty)$ and, since $\overline{e^{t \sigma\left(G^{0}\right)}} \subset \overline{e^{t \sigma(G)}}$ we conclude the proof.

\section{Conjugation}

We say that the linear equation (4.1) is hyperbolic if there exists a $t_{0}>0$ such that the linear operator $T\left(t_{0}\right)$ is hyperbolic, that is, its spectrum is disjoint from the unit circle in the complex plane.

The following proposition is an extension to our context of the corresponding results for the phase space $C$ and we ommit the proof since it is analogous to the one given in [5] and [6]. To prove the last statement, we use Theorem 4.3 (iii).

Proposition 5.1. The equation (4.1) is hyperbolic if and only if for any $t \neq 0, T(t)$ is hyperbolic. In this case, there exist closed linear subspaces $L^{s}$ and $L^{u}$ of $X \times L_{p}$ such that

(i) $L^{s} \oplus L^{u}=X \times L_{p}$;

(ii) $L^{s}$ and $L^{u}$ are invariant under the flow;

(iii) There exist positive constants $\alpha$ and $K$ such that

$$
\begin{aligned}
& \|T(t)(\xi, \varphi)\| \leq K e^{-\alpha t}\|(\xi, \varphi)\|, \quad(\xi, \varphi) \text { in } L^{s}, \quad t \geq 0 \\
& \|T(t)(\xi, \varphi)\| \leq K e^{\alpha t}\|(\xi, \varphi)\|, \quad(\xi, \varphi) \text { in } L^{u}, \quad t \leq 0
\end{aligned}
$$

(iv) $L^{s}$ and $L^{u}$ are characterized by:

$$
\begin{aligned}
& L^{s}=\left\{(\xi, \varphi) \in X \times L_{p}: T(t)(\xi, \varphi) \rightarrow 0 \text { as } t \rightarrow+\infty\right\} \\
& L^{u}=\left\{(\xi, \varphi) \in X \times L_{p}: T(t)(\xi, \varphi) \rightarrow 0 \text { as } t \rightarrow-\infty\right\}
\end{aligned}
$$

$L^{s}$ is the stable subspace and $L^{u}$ is the unstable subspace of equation (4.1).

(v) If $p=2$, the hyperbolicity of equation (4.1) is equivalent to the existence of a positive number $\delta$ such that $\operatorname{det} \Delta(\lambda) \neq 0$ if $|\operatorname{Re} \lambda|<$ $\delta$.

Suppose that equation (4.1) is hyperbolic. We will show that the flows $\{S(t)\}$ of equation (3.2) and $\{T(t)\}$ of equation (4.1) are conjugates if $\mathcal{D}-D$ and $F-L$ are small enough. 
Given $\varepsilon>0$, let $E_{\varepsilon}: X \times X \rightarrow X, f_{\varepsilon}: X \times X \rightarrow X$ and $g_{\varepsilon}: X \rightarrow X$ be any bounded $C^{1}$-maps, with

$$
\sup _{\left(p_{0}, p_{r}\right) \in X \times X}\left\{\left\|D E_{\varepsilon}\left(p_{0}, p_{r}\right)\right\|,\left\|D f_{\varepsilon}\left(p_{0}, p_{r}\right)\right\|,\left\|D g_{\varepsilon}\left(p_{0}\right)\right\|\right\}<\varepsilon .
$$

A homeomorphism $h: E \rightarrow E$ is said to bc a conjugation for two given maps $T: E \rightarrow E$ and $\mathcal{S}: E \rightarrow E$ if $T h=h \mathcal{S}$.

Lemma 5.2. Let $T \in \mathcal{L}(E)$ be a hyperbolic isomorphism. Then, there exists a $\delta>0$ such that for any perturbation $T+\Phi$ of $T$ by a bounded map $\Phi: E \rightarrow E$ with $\operatorname{Lip} \Phi<\delta$, there exists a unique continuous map $h: E \rightarrow E$ such that $h-I$ is bounded and $T h=h(T+\Phi)$. Furthermore, $h^{-1}$ is continuous, so that $h$ is a conjugation between $T$ and $T+\Phi$.

The proof is an application of the contraction principle. See [12] for details.

Theorem 5.3. Suppose equation (4.1) is hyperbolic with $B(\theta)=$ $a(\theta) M$, where $M \in \mathcal{L}(X)$ is constant and a satisfies the hypothesis (H3). Then, there exists $\varepsilon>0$ such that if $E\left(p_{0}, p_{r}\right)=A_{0} p_{0}+$ $A_{r} p_{r}+E_{\varepsilon}\left(p_{0}, p_{r}\right), f\left(p_{0}, p_{r}\right)=B_{0} p_{0}+B_{r} p_{r}+f_{\varepsilon}\left(p_{0}, p_{r}\right)$ and $g=M+g_{\varepsilon}$, for any $p_{0}$ and $p_{r}$ in $X$, then, there exists a unique homeomorphism $h: X \times L_{p} \rightarrow X \times L_{p}, h-I$ bounded, such that $h$ is a conjugation between the flows $\{\mathcal{S}(t)\}$ and $\{T(t)\}$.

Proof: We note that for $\varepsilon$ less than $\left\|A_{0}^{-1}\right\|^{-1}$ and $\left\|A_{r}^{-1}\right\|^{-1}$ the hypotheses (H1) and (H2) are verified for $E, f, g$ and the flow $\{S(t)\}$ is well defined.

Let us write equation (3.2) into the form

$$
D x_{t}=\xi+\int_{0}^{t} L x_{s} d s+Q(t)
$$

where $Q(t) \triangleq-E_{\varepsilon}(x(t), x(t-r))+\int_{0}^{t}\left[f_{\varepsilon}(x(s), x(s-r))+\int_{-r}^{0} a(\theta) g_{\varepsilon}(x(s+\right.$ $\theta)) d \theta] d s$, with $x_{t}=\pi_{2} S(t)(\xi, \varphi)$.

We now define $\Phi_{\ell}: X \times L_{p} \rightarrow X \times L_{p}$ by $\mathcal{S}(t)=T(t)+\Phi_{t}$.

The map $\Phi_{t}$, satisfies:

$$
\begin{aligned}
\pi_{1} \Phi_{t}(\xi, \varphi)= & \int_{0}^{t}\left[f_{\varepsilon}(x(s), x(s-r))+\int_{-r}^{0} a(\theta) g_{\varepsilon}(x(s+\theta)) d \theta\right] d s \\
& +\int_{0}^{t}\left[B_{0} z(s)+B_{r} z(s-r)+\int_{-r}^{0} B(\theta) z(s+\theta) d \theta\right] d s \\
\pi_{2} \Phi_{t}(\xi, \varphi)= & z_{t}
\end{aligned}
$$


where $z_{t}=K(t, 0) Q_{\mid[0, t]}\left(K(t, 0) Q_{\mid[t, 0]}\right.$ if $\left.t<0\right)$ is the solution of $D z_{t}=$ $\int_{0}^{t} L z_{s} d s+Q(t)$ with $z_{0}=0$.

With some laborious but straightforward work, we can prove that $\Phi_{t}$ is continuous, bounded and $\Phi_{r}$ is Lipschitzian with $\operatorname{Lip} \Phi_{T} \leq \frac{c_{1} \varepsilon}{1-c_{2} \varepsilon}, c_{1}$ and $c_{2}$ are constants dependent only on equation (4.1).

We now apply Lemma 5.2 to the hyperbolic operator $T(r)$ : we take $\varepsilon>0$ such that $\operatorname{Lip} \Phi_{r}<\delta$, so that $T(r)$ and $\mathcal{S}(r)$ are conjugated by a unique homeomorphism $h$ of $X \times L_{p}, h-I$ bounded.

For any $t$ in $(-\infty, \infty)$, we define $h_{t}: X \times L_{p} \rightarrow X \times L_{p}$ by $h_{t}=$ $T(-t) h S(t)$.

Clearly, $h_{t}$ is continuous, $\left\|h_{t}-I\right\| \leq\|T(-t)\|\left(\|h-I\|+\left\|\Phi_{t}\right\|\right)<\infty$ and $T(-r) h_{t} S(r)=T(-t-r) h S(t+r)=T(-t) h S(t)=h_{t}$, so that $h_{t}$ also conjugates $S(r)$ and $T(r)$. By the uniqueness in Lemma. $5.2, h_{t}=h$ and $T(t) h=h S(t)$ for all $t$.

We note that, in the above theorem, it is not necessary that equation (4.1) be the linearization of equation (3.2) around a constant solution.

Corresponding to a constant solution $x(t) \equiv c$ of equation (3.2), there is a fixed point $(E(c, c), c)$ of the flow $\mathcal{S}(t)$. In this case, $f(c, c)+\int_{-r}^{0} a(\theta) d \theta g(c)$ must be zero, and conversely.

We say that such a fixed point is hyperbolic if the linearized equation around it is hyperbolic.

The proof of the next Lemma can be found in [4].

Lemma 5.4. Let $X$ be a Hilbert-space, $l \geq 1$ an arbitrary integer, and $G: X^{l} \rightarrow X$ an application of class $C^{1}$ with $G(0)=0, G^{\prime}(0)=0$. Then, for any $\varepsilon>0$, there exist $\delta>0$ and $G_{\varepsilon}: X^{i} \rightarrow X, G_{\varepsilon}$ of class $C^{1}$, such that $G_{\varepsilon}(q)=G(q)$ for every $q \in X^{l}$ with $\|q\| \leq \delta$ and $\sup _{q \in X^{l}}\left\|G_{\varepsilon}(q)\right\|<$ $\varepsilon, \sup _{q \in X^{\prime}}\left\|G_{\varepsilon}^{\prime}(q)\right\|<\varepsilon$.

Theorem 5.5. Suppose that $(0,0) \in X \times L_{p}$ is a hyperbolic fixed point of equation (3.2) and equation (4.1) is the linearized equation around $(0,0)$. Then, there exists a homeomorphism $h: X \times L_{p} \rightarrow X \times L_{p}, h-I$ bounded, such that for each $\tau>0$, there exists $\delta>0$ such that if $(\xi, \varphi)$ is in $X \times L_{\infty}$ and $\|(\xi, \varphi)\|_{X \times L_{\infty}}<\delta$ then $T(t) h(\xi, \varphi)=h S(t)(\xi, \varphi)$ for any $t$ in $[-\tau, \tau]$.

Proof: Let $\widetilde{E}=E-D E(0,0), \widetilde{f}=f-D f(0,0)$ and $\widetilde{g}=g-D g(0)$. For a given $\varepsilon>0$, we apply Lemma 5.4 to obtain $\delta_{1}>0$ and bounded $C^{1}$-functions $E_{\varepsilon}, f_{\varepsilon}$ and $g_{\varepsilon}$ such that $E_{\varepsilon}\left(p_{0}, p_{r}\right)=\tilde{E}\left(p_{0}, p_{T}\right), f_{\varepsilon}\left(p_{0}, p_{r}\right)=$ $\widetilde{f}\left(p_{0}, p_{r}\right)$ and $g_{\epsilon}\left(p_{0}\right)=\widetilde{g}\left(p_{0}\right)$ for $\left|p_{0}\right|$ and $\left|p_{r}\right|$ less than $\delta_{1}$ and relation (5.1) holds. 
We choose $\varepsilon$ sufficiently small and apply Theorem 5.3 to the equation (4.1) and the perturbed equation defined by the maps $D E(0,0)+$ $E_{\varepsilon}, D f(0,0)+f_{\varepsilon}$ and $D g(0)+g_{\varepsilon}$ (with the same kernel $a(\theta)$ ). Let $\{\widetilde{\mathcal{S}}(t)\}$ be the flow of this perturbed equation. We get a homeomorphism $h$ of $X \times L_{p}, h-I$ bounded, such that $T(t) h=h \widetilde{\mathcal{S}}(t)$ for all $t$.

Since $X \times L_{\infty}$ is invariant under $\widetilde{\mathcal{S}}(t)$, using the continuity of the solution with respect to $(\xi, \varphi)$ in $X \times L_{\infty}$, for a given $\tau>0$ we can find $\delta>0$ such that if $\|(\xi, \varphi)\|_{X \times L_{\infty}}<\delta$, then $|x(t+\theta)|<\delta_{1}$ a.e. for $t$ in $[-\tau, \tau]$ and $\theta$ in $[-r, 0]$, where $x_{t}=\pi_{2} \widetilde{S}(t)(\xi, \varphi)$.

Since in the above situation $\widetilde{\mathcal{S}}(t)$ and $\mathcal{S}(t)$ coincide, the proof is complete.

\section{References}

1. Borisovic, J. C. And Turbabin, A. S., On the Cauchy problem for linear homogeneous equations with retarded argument, Dokl. Acad. Nauk. S.S.S.R. 4 (1969).

2. Chow, S. N. And Hale, J. K., "Methods of bifurcation theory," Springer-Verlag, 1982.

3. Dunford, N. And Schwartz, J. J., "Linear operators, Part 1 ," Interscience, New York, 1958.

4. Fichman., L., Equações diferenciais funcionais neutras com condiçôes iniciais descontínuas, Doctoral Thesis, IME-USP (1991).

5. HALE, J. K., "Theory of functional differential equations," Springer-Verlag, Berlin, 1977.

6. HENRY, D., Linear autonomous neutral functional differential equations, J. Diff. Equations 15 (1974).

7. Henry, D., A property of the exponential function, Publicacions Matemàtiques U.A.B. 31(1) (1987), Bellaterra.

8. Henry, D., A difference equation, personal communication, Universidade de São Paulo (1989).

9. Henry, D., Derivadas de Gâteaux. Hadamard e Fréchet, personal communication, Lniversidade de São Paulo (1990).

10. Herbst, I., The spectrum of Hilbert space semigroups, J. Operator Theory 10 (1983).

11. KRASNOSEL'SKII, M. A., "Topological methods in the theory of nonlinear integral equations," The Macmillan Company, New York, 1964. 
12. NITECKI, Z., "Differentiable dynamics; an introduction to the orbit structure of diffeomorphisms," The M.I.T. Press, Cambridge, Mass., 1971.

13. PAlis JR., J., Seminário de Sistemas Dinâmicos, IMPA (1971).

Instituto de Matemática e Estatística

Universidade de São Paulo

01498 - São Paulo (São Paulo (SP))

BRASIL

Rebut el 30 d'Abril de 1993 